\title{
Distribution, characterization, and antibiotic resistance of hypervirulent Klebsiella pneumoniae isolates in a Chinese population with asymptomatic bacteriuria
}

\author{
Jun Li ${ }^{1,2}$, Yanbing Li ${ }^{1}$, Mengli Tang ${ }^{1}$, Fengjun Xia ${ }^{1}$, Changhang Min ${ }^{1}$, Yongmei Hu ${ }^{1,2}$, Haichen Wang ${ }^{1,2}$, \\ Jingyi Zhang ${ }^{1}$ and Mingxiang Zou ${ }^{1,2^{*}}$
}

\begin{abstract}
Background: Asymptomatic bacteriuria (ASB) frequently occurs among all ages and may develop into urinary tract infections (UTIs). Hypervirulent Klebsiella pneumoniae (hvKP) has become a new threat to human health. In our study, we aimed to investigate the epidemiological characteristics of hvKP in population with ASB.

Results: A total of 61 K. pneumoniae isolates were collected from 7530 urine samples between October and December 2020. The strains were sensitive to most of the antimicrobial agents tested, but a polymyxin resistant strain was found $(\mathrm{MIC}>16 \mu \mathrm{g} / \mathrm{mL})$. Three serotypes were detected, including K1 $(16.4 \%, 10 / 61), \mathrm{K} 5$ (1.6\%, 1/61) and K57 (3.2\%, 2/61). Four strains (KPNY9, KPNY31, KPNY40, and KPNY42) carried a combination of two or more hypervirulent markers (peg-344, iroB, iucA, $r m p A$, and $p^{r m p A 2)}$, and their survival rates after Galleria mellonella infection were lower than those of the other strains (40.0 vs. 70.0\%), suggesting that they were hvKP. These hvKP strains with lower biofilm forming ability than classical $K$. pneumoniae $(0.2625 \pm 0.0579$ vs. $0.6686 \pm 0.0661, P=0.033)$ were identified as belonging to K2-ST65, K2-ST86, K57-ST592, and K2-ST5559 (a new ST type). KPNY31 (ST5559) shared a close genetic relationship with KPNY42 (ST86) and other ST86 isolates, which have been detected in both nosocomial and community-acquired infections.

Conclusions: The hvKP with relatively weak biofilm formation was detected in a population with ASB, which was more likely to cause bacteremia and serious consequences. A novel sequence type (ST5559) hvKP derived from ST86 was found. Therefore, hvKP should be monitored in the population with ASB.

Keywords: Hypervirulent K. pneumoniae, Asymptomatic bacteriuria, Antimicrobial susceptibility, Virulence-associated genes, Biofilm formation, Whole-genome sequencing (WGS)
\end{abstract}

\section{Background}

Urinary tract infections (UTIs), which affect 150 million people each year, have emerged as one of the most common bacterial infections that affect people of all ages and

*Correspondence: zoumingxiang@csu.edu.cn

${ }^{1}$ Department of Clinical Laboratory, Xiangya Hospital, Central South

University, Changsha 410008, Hunan, China

Full list of author information is available at the end of the article genders [1]. The diagnosis requires a combination of relevant signs, symptoms, and a positive urine culture (UC). Positive urine culture without associated signs and symptoms is defined as asymptomatic bacteriuria (ASB) [2]. ASB becomes more common with age, from $<2 \%$ in children to up to $50 \%$ in elderly residents of long-term care facilities [2]. 
ASB is frequently caused by the same types of bacteria as in symptomatic UTIs, such as Escherichia coli, Klebsiella pneumoniae, Enterococcus faecium, Enterococcus faecalis, and so on, among which $E$. coli and $K$. pneumoniae are mainly predominant [3]. Hypervirulent $K$. pneumoniae (hvKP) is a variant of $K$. pneumoniae (KP), but more pathogenic, easily transmitted, and with a higher mortality rate $[4,5]$. It can cause multiple sites of infection, including abdominal disease, thoracic disease, endophthalmitis, central nervous system disease, and genitourinary tract infections [5]. Recently, it has also been detected in UTIs with multidrug resistance and even extensively drug-resistant isolates [6-8]. Worse yet, hvKP infection was more likely to cause bacteremia and serious consequences than classical K. pneumoniae (cKP) when it developed into UTIs [9].

However, studies on hvKP in ASB are still rare [10]. Therefore, our study investigated the molecular epidemiological characteristics of hvKP in ASB to provide guidelines for the empirical treatment of UTIs.

\section{Results}

\section{Characteristics of cKP and hvKP isolates}

A total of 7530 urine samples were collected and $61 \mathrm{~K}$. pneumoniae isolates were found from 61 people, 20 men and 41 women, respectively. Galleria mellonella has long been used as an infection model for studying bacterial and fungal infections, as well as evaluating the efficacy of new antimicrobial drugs. In our current study, using the G. mellonella infection model, we investigated the virulence of the strains and discovered that the survival rates after infection with four strains (KPNY9, KPNY31, KPNY40, and KPNY42) were less than 40.0 percent. In contrast, the other isolates in our study had a survival rate of more than 70.0 percent (Fig. 1). Furthermore, the four strains had hypermucoviscosity, which belonged to $\mathrm{K} 2$ or $\mathrm{K} 57$, and co-carried two or more hypervirulent markers (peg-344, iroB, iucA, ${ }_{p} r m p A$, and ${ }_{p} r m p A 2$ genes), indicating that they were hvKP (Fig. 2).

There was no significant difference in basic subjects' demographics between the cKP group and the hvKP group, such as age, sex, white blood cell (WBC) count, and nitrite. The detailed baseline characteristics of the enrolled participants are summarized in Table 1.

The $b l a_{\mathrm{SHV}-28}$, fosA5-like, $o q x A$, and $o q x B$ genes were detected in KPNY31 and KPNY42. The $b l a_{\mathrm{SHV}-11}$, $b l a_{\mathrm{SHV}-67}, f o s A 5$-like, $o q x A$ and $o q x B$ genes were present in KPNY9, while $b l a_{\mathrm{SHV}-26}, f o s A 5, o q x A$, and $o q x B$ were detected in KPNY40 (Fig. 2)

\section{Antimicrobial susceptibility of $K$. pneumoniae isolates}

We tested the sensitivity of the isolated KP strains to a panel of antimicrobial agents and discovered that all strains were sensitive to the most of agents detected. The sensitivity rates of the $61 \mathrm{KP}$ isolates to ceftazidime (CAZ), cefepime (FEP), aztreonam (ATM), nitrofurantoin (NIT), and levofloxacin (LVX) were 96.7\% (59/61), 91.8\% (56/61), 95.1\% (58/61), 98.4\% (60/61), and 98.4\%

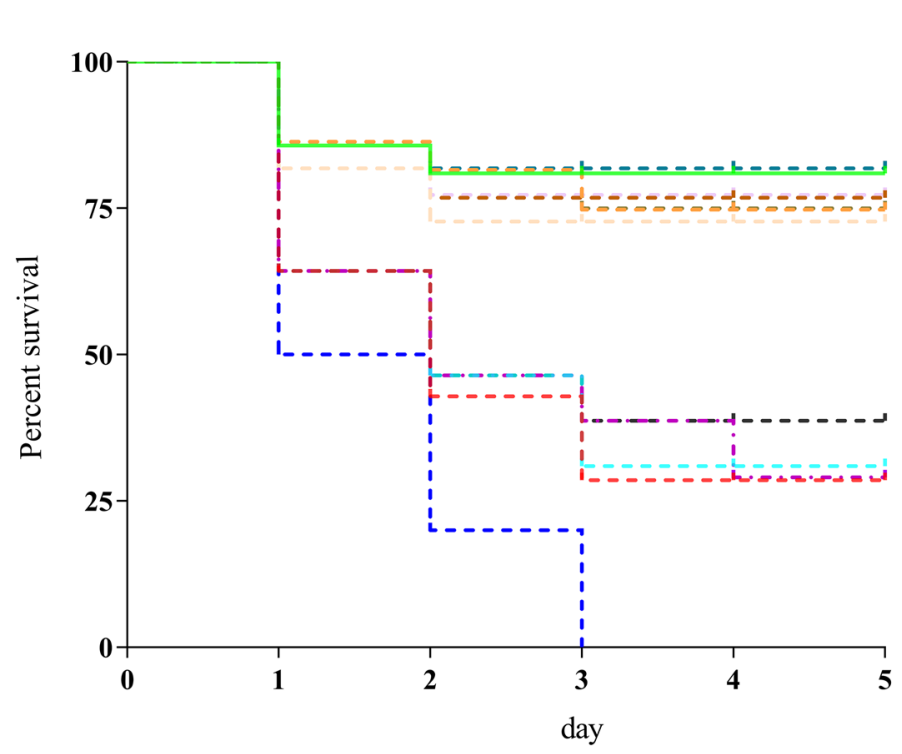

+ ATCC 700603

- ᄂ. NTUH-K2044

- ᄂ. KPNY31

- ᄂ. KPNY9

-'- KPNY40

- ᄂ. KPNY42

KPNY5

-ᄂ. KPNY11

- ᄂ. KPNY26

ㄴ. KPNY33

- ᄂ. KPNY48

- ᄂ. KPNY52

ulence of KP isolates. The virulence of $1 \times 10^{6} \mathrm{CFU}$ of each KP strain (KPNY9, KPNY31, KPNY40, KPNY42, KPNY5, KPNY11, KPNY26, KPNY33, KPNY48, and KPNY52) was investigated using a G. mellonella infection model. ATCC 700603 and NTUH-K2044 were used as low and high virulence KP controls, respectively. The four isolates (KPNY9, KPNY31, KPNY40, KPNY42) resulted in significantly lower survival rates than ATCC 700603, suggesting that they were hypervirulent 


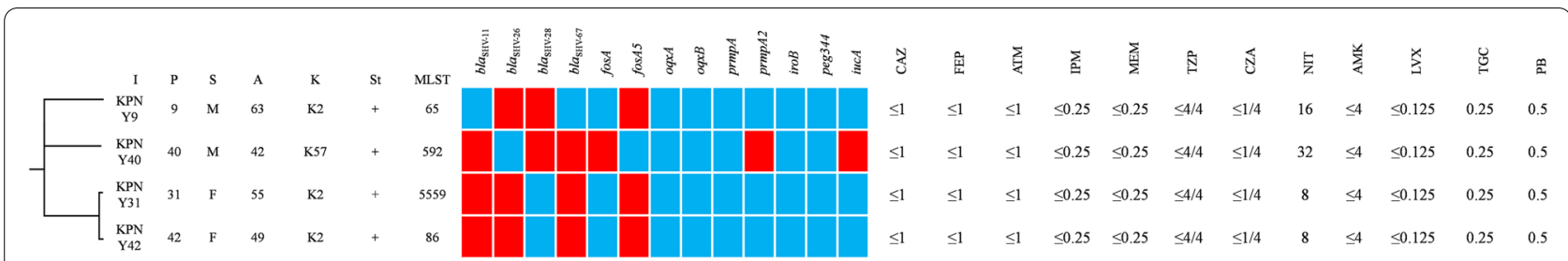

Fig. 2 Clinical characteristics, antimicrobial susceptibility, resistance genes, and virulence-associated genes of four hvKP isolates. I, isolate; P, patient; $\mathrm{S}$, sex; A, age; K, K serotype; St, string test; CAZ, ceftazidime; FEP, cefepime; IPM, imipenem; MEM, meropenem; CZA, ceftazidime / avibactam; ATM, aztreonam; TZP, piperacillin / tazobactam; NIT, nitrofurantoin; AMK, amikacin; LVX, levofloxacin; TGC, tigecycline; PB, polymyxin. The red and blue squares indicate the absence and presence of the genes indicated at the top, respectively

Table 1 Comparison of clinical characteristics and antimicrobial susceptibility patterns between hvKP and cKP isolates

\begin{tabular}{llll}
\hline Clinical characteristic & hvKP $(\boldsymbol{n}=\mathbf{4})$ & $\mathbf{c K P}(\boldsymbol{n}=\mathbf{5 7})$ & $\boldsymbol{P}$ value \\
\hline Basic demographics & & & \\
Age & $52.25 \pm 4.46$ & $42.72 \pm 1.715$ & 0.154 \\
Male & $2(50.0 \%)$ & $18(31.6 \%)$ & 0.835 \\
WBC (0) & $4(100.0 \%)$ & $57(100.0 \%)$ & - \\
Nitrite (negative) & $4(100.0 \%)$ & $57(100.0 \%)$ & - \\
Antimicrobial susceptibility [n (S, \%)] & & \\
Ceftazidime & $4(100.0 \%)$ & $53(93.0 \%)$ & 1.000 \\
Cefepime & $4(100.0 \%)$ & $52(91.2 \%)$ & 1.000 \\
Imipenem & $4(100.0 \%)$ & $57(100.0 \%)$ & - \\
Meropenem & $4(100.0 \%)$ & $57(100.0 \%)$ & - \\
Ceftazidime / avibactam & $4(100.0 \%)$ & $57(100.0 \%)$ & - \\
Aztreonam & $4(100.0 \%)$ & $52(91.2 \%)$ & 1.000 \\
Piperacillin / tazobactam & $4(100.0 \%)$ & $57(100.0 \%)$ & - \\
Nitrofurantoin & $2(50.0 \%)$ & $46(80.7 \%)$ & 0.196 \\
Amikacin & $4(100.0 \%)$ & $57(100.0 \%)$ & - \\
Levofloxacin & $4(100.0 \%)$ & $53(93.0 \%)$ & 1.000 \\
Tigecycline & $4(100.0 \%)$ & $57(100.0 \%)$ & - \\
Polymyxin & $4(100.0 \%)$ & $56(98.2 \%)$ & 1.000 \\
\hline
\end{tabular}

$n$, number, $S$ sensitive, - not determined

(60/61), respectively; the strains demonstrated $100.0 \%$ sensitivity to ceftazidime / avibactam (CZA), piperacillin / tazobactam (TZP), imipenem (IPM), meropenem (MEM), amikacin (AMK), and tigecycline (TGC). One strain was resistant to polymyxin but susceptible to all other antimicrobial agents (except for NIT). There was no significant difference in drug sensitivity between hvKP and cKP isolates in our study (Table 1).

\section{Virulence factors}

Four serotypes were identified among the $61 \mathrm{KP}$ isolates: $\mathrm{K} 1, \mathrm{~K} 2, \mathrm{~K} 5$, and $\mathrm{K} 57$. In addition, K2 and K57 serotypes were detected in hvKP strains, with a predominance of K2 serotypes (3/4). In 57 cKP isolates, three serotypes were found: $K 1, K 5$, and $K 57$, with rates of $17.5,1.8$, and
Table 2 Comparison of virulence factors between hvKP and cKP isolates

\begin{tabular}{|c|c|c|c|}
\hline Virulence factors & $\operatorname{hvKP}(n=4)$ & $\operatorname{cKP}(n=57)$ & $P$ value \\
\hline \multicolumn{4}{|l|}{ K serotype } \\
\hline K1 & 0 & $10(17.5 \%)$ & 1.000 \\
\hline K2 & $3(75.0 \%)$ & 0 & 0.000 \\
\hline $\mathrm{K} 5$ & 0 & $1(1.8 \%)$ & 1.000 \\
\hline K57 & $1(25.0 \%)$ & $2(3.5 \%)$ & 0.187 \\
\hline hypermucoviscosity & $4(100.0 \%)$ & $4(7.0 \%)$ & 0.000 \\
\hline$p^{r m p A}+i r o B+p e g-344$ & $1(25.0 \%)$ & 0 & 0.066 \\
\hline $\begin{aligned} & p^{r m p A}+{ }_{p} r m p A 2+i u c A \\
+ & i r o B+p e g-344\end{aligned}$ & $3(75.0 \%)$ & 0 & 0.000 \\
\hline
\end{tabular}

$3.5 \%$, respectively. Four of the hvKP isolates and four of the $57 \mathrm{cKP}$ isolates had hypermucoviscosity. When compared to cKP isolates, hvKP isolates had higher rates of $\mathrm{K} 2$ and prmp $A+$ prmpA $2+i u c A+i r o B+p e g-344$. Furthermore, the hypermucoviscous phenotype was more prevalent in hvKP isolates (Table 2).

\section{Biofilm formation}

We investigated the biofilm formation of KP isolates and discovered that all KP isolates were biofilm producers, albeit to varying degrees: strong biofilm producers $(63.9 \%, 39 / 61)$, medium biofilm producers $(24.6 \%, 15 / 61)$, and weak biofilm producers $(11.5 \%, 7 / 61)$. In 1,2 , and 1 hvKP isolates, strong biofilm, medium biofilm, and weak biofilm formation were observed, respectively (Fig. 3A). KPNY31 had a moderate ability to form biofilms. There were $38 \mathrm{cKP}$ strains with strong biofilm formation, 13 with medium biofilm formation, and 6 with weak biofilm formation among the cKP strains (Fig. 3B). cKP was more capable of forming biofilms than hvKP $(0.6686 \pm 0.0661$ vs. $0.2625 \pm 0.0579, P=0.033$ ) (Fig. 3 C).

\section{Phylogenic analysis}

The results of sequencing showed that among the four hvKP strains (KPNY9, KPNY31, KPNY40, and KPNY42), KPNY31 and KPNY42 were more closely related, and the 
A

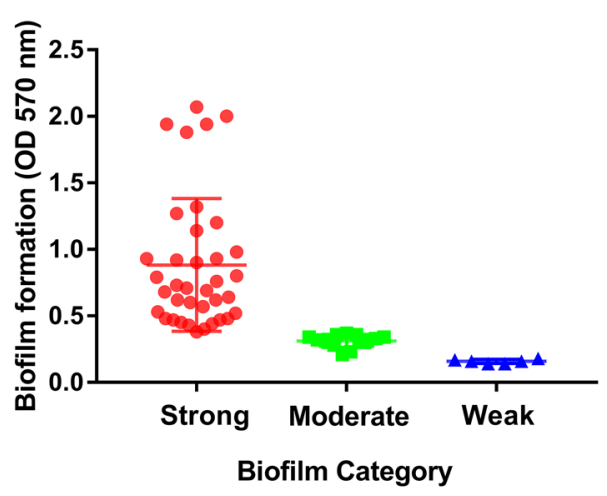

B

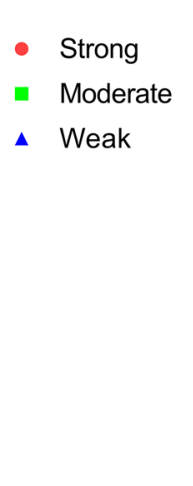

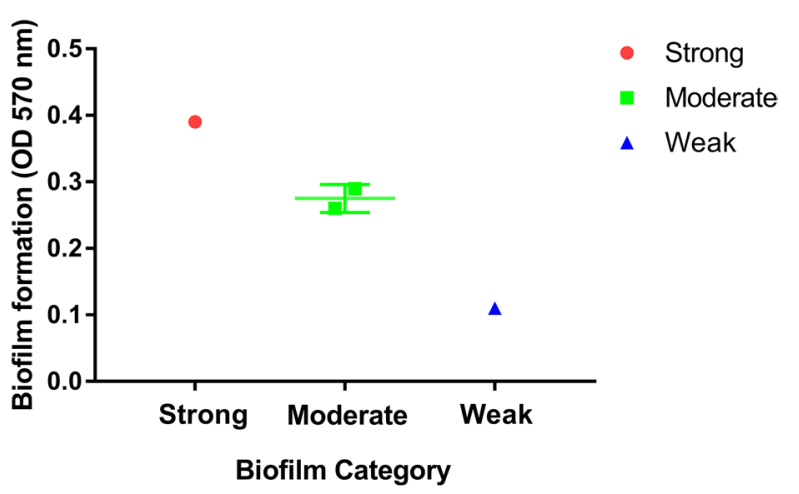

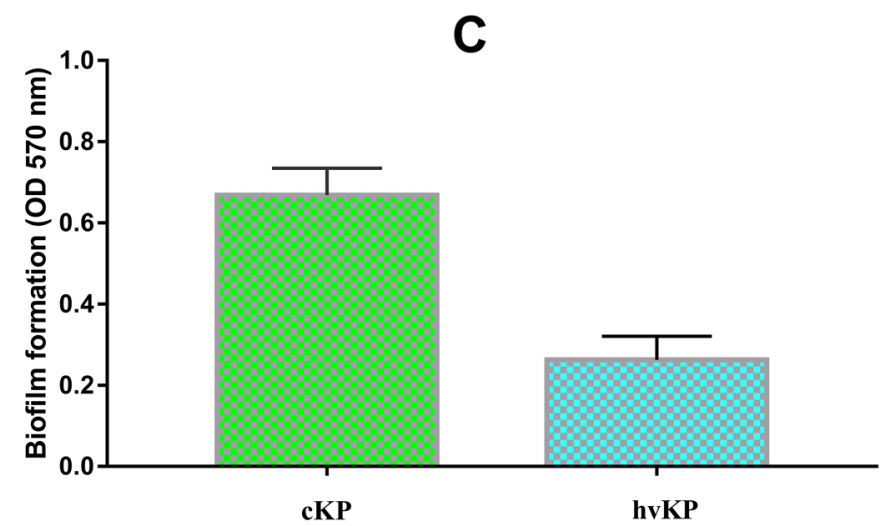

Fig. 3 Biofilm formation of hvKP and cKP. Biofilm formation was determined using crystal violet assay in 96-well plates. A Quantification and categorization of biofilm formation of cKP isolates. B Quantification and categorization of biofilm formation of hvKP isolates. C Biofilm formation of hvKP and CKP was compared; there was a significant difference in the biofilm formation between hvKP and cKP $(0.2625 \pm 0.0579$ vs. $0.6686 \pm$ $0.0661, P=0.033)$

remaining two strains were more distantly related (Fig. 2). In addition, MLST analysis revealed that the four hvKP strains were classified as ST65, ST86, ST592, and ST5559 (9-4-2-363-1-1-27, a new ST type). ST5559 hvKP's resistance pattern, set of resistant genes, and virulence-related genes were identical to those of ST86's KPNY42 (Fig. 2). BacWGSTdb 2.0 was used to perform phylogenetic analysis on the ST5559 isolate (KPNY31) with the following parameters: SNP threshold of 200, MLST scheme of cgMLST, and MLST threshold of 500. Using the cgMLST strategy, a total of 55 closely related isolates were identified, all of which belonged to ST86.

KPNY31 (ST5559) belonged to group A of the isolates, which were divided into four groups (A-D). SA1 (accession number: CBTW01), a hvKP isolated from France, was the most closely related isolate to KPNY31, with 67 different alleles (Fig. 4.).

\section{Discussions}

ASB frequently occurs among all ages with the possibility of developing into UTIs. HvKP has become a new threat to human health and has also been reported in UTIs with multidrug resistance and even extensively drug-resistant isolates. However, the epidemiological characteristics of hvKP in a population with ASB were uncovered. This study examined hvKP isolated from population with ASB at our hospital from October to December 2020. Four hvKP strains were detected from $61 \mathrm{KP}$ isolates, with a rate of $6.6 \%$. The detection rate was lower than that of Zafar et al. reported in complicated urinary tract infections in kidney stone patients with $29.5 \%(18 / 61)$, and Taraghian et al. reported in urinary tract infections with $10.5 \%(11 / 105)[6,11]$.

In this study, all $61 \mathrm{KP}$ strains were sensitive to most of the tested antibiotics. It is worth noting that one strain showed resistance to polymyxin. Polymyxin has been used as an important antibiotic in the treatment of multidrug-resistant bacterial infections. However, 


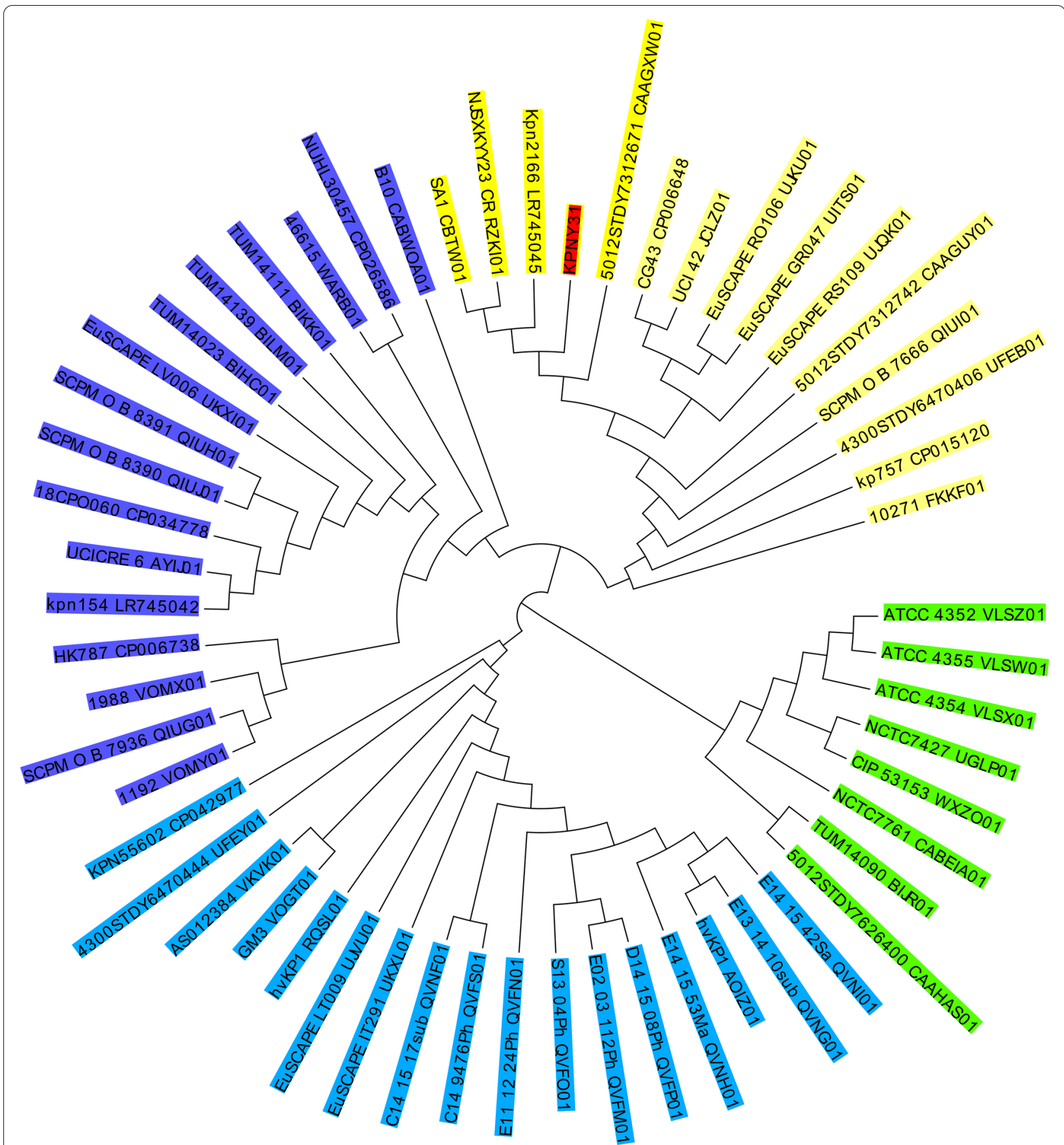

Fig. 4 The phylogenetic analysis of ST5559. Phylogenic analysis was performed using BacWGSTdb 2.0 with the following parameters: SNP threshold of 200, MLST scheme with cgMLST and MLST threshold of 500. The isolates were divided into four groups: A (yellow), B (dark blue), C (light blue), and $\mathbf{D}$ (green). ST5559 belonged to group A

due to its widespread clinical use, polymyxin-resistant strains are becoming more prevalent because of the plasmid-mediated $\mathrm{mor}$ gene, and polymyxin-resistant hvKP strains have emerged $[12,13]$. In addition, antibiotics are widely used in animals for disease prevention and treatment, which promotes the emergence of polymyxin-resistant strains [14], and humans can acquire drug-resistant strains through diet and other routes.

We examined virulence-related genes in these strains; eight strains were string test-positive, while four strains 
were identified as hvKP by G. mellonella larvae, indicating that not all string test-positive strains were hvKP, which is consistent with some previous reports [15]. Three K2 strains and one K57 strain were hvKP, while all K1 serotype strains were non-hvKP. Six strains carried two or more virulence-related genes simultaneously. Four of these isolates were identified as hvKP, indicating that isolates carrying multiple virulence genes at the same time considerably enhanced the chance of hvKP strains.

Previous research has linked the K2 serotype of hvKP to a broader range of STs, such as ST25, ST65, ST66, and ST86 [16-19], which have been detected not only in nosocomial infections but also in community-acquired infections [20-23]. These results indicate that these ST types of hvKP may be transmitted both within and outside of hospitals. In our study, two K2 serotype hvKP strains belonging to ST65 and ST86 were detected in a population with ASB, suggesting that ST65 and ST86 hvKP strains could be an important reservoir for UTIs. Another K2 serotype hvKP strain was identified to belong to ST5559 (a new ST type). Notably, the resistance pattern, set of resistant genes, and virulence-related genes of the ST5559 hvKP were identical to KPNY42, which belongs to ST86. Furthermore, compared with other isolates using BacWGSTdb 2.0, the strain had a close genetic relationship with SA1 (accession number: CBTW01) with 67 different alleles (ST86), which was hvKP from Paris, France, illustrating that the strain could have evolved from ST86 but the specific communication mechanism is not precise. In addition to the K2 serotype hvKP, K57 was also detected in our study; this serotype has been reported in other regions of China [24] and Japan [25]. To the best of our knowledge, this is the first study revealing that K57 hvKP belongs to ST592.

Biofilm formation plays an important role in the invasion of KP into bladder epithelial cells as well as the escape of phagocytic phagocytosis in UTIs [26]. Our study showed that all hvKP had biofilm formation ability, and there was no difference in the detection rate of hvKP and cKP. Cubero et al. reported that hvKP could form robust biofilms at the air-liquid interface [27]. However, this finding was inconsistent with the report of Zafar et al., which showed that the biofilm formation ability of hvKP was higher than that of cKP from complicated urinary tract infection in kidney stone patients (94.4 vs. $86.0 \%$ ) [6]. These conflicting results may be related to the different subject groups. All subjects in this study were the population with ASB. Of course, this may also be related to only four hvKP strains detected in this study, and the few strains may not be representative. Although all hvKP isolates in our study produced biofilm, the optical density (OD) value was significantly lower than that of cKP, suggesting that the biofilm-forming ability of hvKP was weaker than that of cKP and these were more likely to cause bacteremia and serious consequences when it developed into UTIs [9].

This study has some limitations. First, the time required to collect strains within several months is relatively brief. Additionally, our study examined a small number of strains. Finally, biomarker profiles and G. mellonella larvae were used to determine hvKP isolates rather than a mouse model.

\section{Conclusions}

Although the majority of the KP isolates identified in our study were susceptible to most antibiotics, a polymyxinresistant strain was found. Notably, hvKP isolates were detected in a population with ASB with lower biofilm formation ability than that of cKP and easily caused systemic infection with serious consequences. This is the first report of ST5559 hvKP, and our results indicate that it has some relationship with ST86 detected in nosocomial infections. Therefore, hvKP strains should be monitored in the population with ASB.

\section{Methods \\ Collection and identification of $K$. pneumoniae isolates}

K. pneumoniae isolates were collected from urine specimens of population with no symptoms of UTIs at Central South University's Xiangya Hospital (Changsha, China) from October to December 2020. Xiangya Hospital is a general hospital with 3,500 beds and manages approximately 3 million patients from all over the country every year. Aseptic operation was used to collect $5-10 \mathrm{~mL}$ of freshly voided midstream urine with sterile and widemouthed plastic bottles with a tight cap. Using calibrated inoculating loop $0.001 \mathrm{~mL}$ of uncentrifuged, uniformly mixed, midstream urine samples were aseptically inoculated onto blood agar (Guangzhou Boret Biotechnology Co., Ltd, China). After overnight incubation at $37^{\circ} \mathrm{C}$ for $24-48 \mathrm{~h}$ colonies were counted to check significant growth. Colony counts yielding bacterial growth of $10^{5}$ / $\mathrm{mL}$ urine were regarded as significant for bacteriuria [2]. All isolates were identified using matrix-assisted laser desorption/ionization time-of-flight mass spectrometry (MALDI-TOF MS; Bruker Daltonics GmbH, Bremen, Germany) with Escherichia coli ATCC 25922 as a quality control strain (National Center for Clinical Laboratories, Beijing, China). Briefly, one colony from an overnight culture was taken with a disposable loop and spotted onto a metal plate and the spots were then covered with $1 \mu \mathrm{L}$ of a $\alpha$-cyano-4-hydroxy-cinnamic acid (HCCA) matrix (Bruker Daltonik GmbH, Bremen, Germany). Then, bacterial samples on the microplate were analyzed with 
MALDI-TOF MS. Finally, MALDI Biotyper ${ }^{\circledR}$ (Bruker Daltonik GmbH, Bremen, Germany) software was used to classify the isolate at the genus and species level.

\section{Identification of hvKP isolates}

The string test was carried out to characterize the hypermucoviscous phenotype [28]. HvKP isolates were screened for the presence of ${ }_{p} r m p A,{ }_{p} r m p A 2$, iroB, peg344, and iucA or any combination of these genes as previously described [15]. The virulence of all the collected isolates was evaluated using an infection model of G. mellonella larvae (Tianjin Huiyude Biotech Company, Tianjin, China) as described previously [29]. Overnight cultures of $K$. pneumoniae were adjusted to $1 \times 10^{8}$ colony forming units (CFU) / $\mathrm{mL}$ using phosphate-buffered saline. G. mellonella larvae were injected with $10 \mu \mathrm{L}$ of the bacterial culture and incubated in the dark at $37^{\circ} \mathrm{C}$ for 5 days; survival was continuously monitored. K. pneumoniae NTUH-K2044 and K. pneumoniae ATCC 700603 were used as high and low virulence control strains, respectively. Experiments were carried out in triplicate.

\section{Antimicrobial susceptibility testing}

The broth microdilution test was used to determine the minimum inhibitory concentrations (MICs) of CAZ, FEP, IPM, MEM, CZA, ATM, TZP, NIT, AMK, LVX, $\mathrm{TGC}$, and $\mathrm{PB}$ in $K$. pneumoniae isolates, which were all purchased from Hangzhou Kangtai Biotechnology Co. The Clinical and Laboratory Standards Institute's (2020) guidelines were used to interpret the susceptibility breakpoints [30]. The MICs of tigecycline were described following the breakpoint established by the US Food and Drug Administration. E. coli ATCC 25922 was used as a quality control strain.

\section{Detection of capsular serotypes}

The genomic DNA of all $K$. pneumoniae strains was extracted from overnight cultured strains using a boiling method [31]. The polymerase chain reaction (PCR) was performed to detect capsular serotype genes (K1, K2, K5, K20, K54, and K57) with primers as previously described [32]. Positive PCR products were subjected to direct Sanger sequencing.

\section{Biofilm formation assay}

The crystal violet staining method was used to test biofilm formation in KP isolates, as described previously [33]. The absorbance at $570 \mathrm{~nm}$ was calculated, and data are presented as the mean \pm standard deviation for each triplicate assay. We used K. pneumoniae ATCC 700606 as a negative control. The OD cut-off (ODc) value was determined using the formula, in accordance with the parameters defined by Ragupathi et al. [34], as follows: ODc $=$ average OD of the negative control $+(3 \times$ standard deviation of negative control). The biofilm formation ability was categorized based on optical density results as follows: (1) strong biofilm producer (OD $>4 \times \mathrm{ODc}$ ); (2) medium biofilm producer $(4 \times$ ODc $\geq$ OD $>2 \times$ ODc); (3) weak biofilm producer $(2 \times$ ODc $\geq$ OD $>$ ODc); and (4) non-biofilm producer (OD $\leq \mathrm{ODc})$.

\section{Whole-genome sequencing (WGS)}

WGS was used to identify resistant genes and virulence factors in hvKP isolates. Approximately $10 \mu \mathrm{g}$ of DNA was extracted from each strain using the DNeasy UltraClean Microbial Kit (QIAGEN, Hilden, Germany) to establish two Illumina paired-end libraries with 500 and 2000 base pairs (average insertion lengths).

The following reads were excluded from the raw data: 1) reads with undefined bases of $5 \mathrm{bp}, 2)$ reads with low-quality ( $\leq$ Q20) bases of $20 \mathrm{bp}, 3$ ) contamination of adapter, and 4) duplicate reads. SOAPdenovo v1.05. was used to assemble the final cleaned reads with genome coverage for each strain $\times 100$ approximately. The CGE server (https://cge.cbs.dtu.dk) was used to identify the resistant genes. The phylogenetic tree was constructed using the BacWGSTdb server using the core genome multilocus sequence typing (cgMLST) and single nucleotide polymorphism (SNP) strategy, as defined previously [35]. The Oxford scheme was used for multilocus sequence typing (MLST), and the sequence types (STs) were allocated through the MLST database (https://bigsdb.paste ur.fr/klebsiella/klebsiella.html). The sequence data were deposited in NCBI with the accession PRJNA745055.

\section{Statistical analyses}

Clinical characteristics, antimicrobial susceptibility, virulence factors, and biofilm formation were compared between the cKP group and the hvKP group. For categorical variables, the $\chi^{2}$ test or Fisher's exact test was used, and for continuous variables, the Student's $t$ test was used. SPSS 21.0 was used for statistical analysis, and $P<$ 0.05 was considered statistically significant.

\section{Abbreviations}

ST: Sequence type; hvKP: Hypervirulent K. pneumoniae; KP: K. pneumoniae; CKP: Classical K. pneumoniae; WBC: White blood cell; MALDI-TOF MS: Matrix-assisted laser desorption/ionization time-of-flight mass spectrometry; CFU: Colony forming units; MIC: Minimum inhibitory concentrations; CAZ: Ceftazidime; FEP. Cefepime; IPM: Imipenem; MEM: Meropenem; CZA: Ceftazidime / avibactam; ATM: Aztreonam; TZP: Piperacillin / tazobactam; NIT: Nitrofurantoin; AMK: Amikacin; LVX: Levofloxacin; TGC: Tigecycline; PB: Polymyxin; PCR: Polymerase chain reaction; OD: Optical density; CgMLST: Core genome multilocus sequence typing; SNP: Single nucleotide polymorphism; MLST: Multilocus sequence typing; WGS: Whole-genome sequencing; UTIs: Urinary tract infections; UC: Urine culture; ASB: Asymptomatic bacteriuria; HCCA: a-cyano-4hydroxy-cinnamic acid. 


\section{Acknowledgements}

We would like to express our gratitude to the staff of the Department of Clinical Laboratory at Central South University's Xiangya Hospital for their assistance in collecting and identifying the K. pneumoniae isolates.

\section{Authors' contributions}

Study design: Jun Li and Mingxiang Zou. Study conduct: Yanbing Li, Fengjun Xia, Changhang Min, Jingyi Zhang and Mengli Tang. Data collection: Mengli Tang, Fengjun Xia, and Changhang Min. Data analysis: Jun Li and Mingxiang Zou. Data interpretation: Yongmei Hu and Haichen Wang. Drafting manuscript: Jun Li. Revising manuscript content: Mingxiang Zou. Approving the final version of the manuscript: Jun Li and Mingxiang Zou. The final manuscript was read and approved by all authors.

\section{Funding}

The National Natural Science Foundation of China (No. 81702068), Natural Science Foundation of Hunan Province (No. 2020JJ4886), the Science Foundation of Hunan Health Commission in Hunan province (No. 202111000066), and the Undergraduate Free Exploration Foundation of Central South University (XCX2021041) supported this study.

\section{Availability of data and materials}

The datasets generated and analyzed during the current study are available from the corresponding author on reasonable request. WGS data appear to have been deposited in NCBI with the accession PRJNA745055.

\section{Declarations}

\section{Ethics approval and consent to participate}

This research was carried out in compliance with the recommendations of the 1964 Helsinki declaration and its later amendments or comparable ethical standards. This study was approved by the Central South University Ethics Committee (Changsha, Hunan Province, China) with ID 201703302. All participants provided written informed consent.

\section{Consent for publication}

Not applicable.

\section{Competing interests}

The authors declare that they have no competing interests.

\section{Author details}

'Department of Clinical Laboratory, Xiangya Hospital, Central South University, Changsha 410008, Hunan, China. ${ }^{2}$ National Clinical Research Center for Geriatric Disorders, Xiangya Hospital, Central South University, Changsha 410008, Hunan, China.

Received: 15 October 2021 Accepted: 6 December 2021

Published online: 18 January 2022

\section{References}

1. Öztürk R, Murt A. Epidemiology of urological infections: a global burden. World J Urol. 2020;38:2669-79.

2. Nicolle LE, Gupta K, Bradley SF, Colgan R, DeMuri GP, Drekonja D, et al. Clinical practice guideline for the management of asymptomatic bacteriuria: 2019 update by the Infectious Diseases Society of America. Clin Infect Dis. 2019;68:e83-e110.

3. Nicolle LE. Asymptomatic bacteriuria and bacterial interference. Microbiol Spectr. 2015;3.

4. Lee CR, Lee JH, Park KS, Jeon JH, Kim YB, Cha CJ, et al. Antimicrobial resistance of hypervirulent Klebsiella pneumoniae: epidemiology, hypervirulence-associated determinants, and resistance mechanisms. Front Cell Infect Microbiol. 2017;7:483.

5. Russo TA, Marr CM. Hypervirulent Klebsiella pneumoniae. Clin Microbiol Rev. 2019;32:e00001-19.

6. Zafar S, Hanif S, Akhtar H, Faryal R. Emergence of hypervirulent $K$. pneumoniae causing complicated UTI in kidney stone patients. Microb Pathog. 2019;135:103647.
7. Mataseje LF, Boyd DA, Mulvey MR, Longtin Y. Two hypervirulent Klebsiella pneumoniae isolates producing a bla $a_{\mathrm{KPC}-2}$ carbapenemase from a Canadian patient. Antimicrob Agents Chemother. 2019;63:e00517-19.

8. Zhu X, Sun C, Chen H, Zhan X, Liu C, Liu X, et al. Co-occurrence of three different plasmids in an extensively drug-resistant hypervirulent Klebsiella pneumoniae isolate causing urinary tract infection. J Glob Antimicrob Resist. 2020;23:203-10.

9. Kim YJ, Kim SI, Kim YR, Wie SH, Lee HK, Kim SY, et al. Virulence factors and clinical patterns of hypermucoviscous Klebsiella pneumoniae isolated from urine. Infect Dis. 2017;49:178-84.

10. Wysocka M, Zamudio R, Oggioni MR, Gołębiewska J, Dudziak A, Krawczyk B. The New Klebsiella pneumoniae ST152 variants with hypermucoviscous phenotype isolated from renal transplant recipients with asymptomatic bacteriuria-genetic characteristics by WGS. Genes. 2020;11:1189.

11. Taraghian A, Nasr Esfahani B, Moghim S, Fazeli H. Characterization of hypervirulent extended-spectrum $\beta$-lactamase-producing Klebsiella pneumoniae among urinary tract infections: the first report from Iran. Infect Drug Resist. 2020;13:3103-11.

12. Lu Y, Feng Y, McNally A, Zong Z. The occurence of colistin-resistant hypervirulent Klebsiella pneumoniae in China. Front Microbiol. 2018:9:2568

13. Huang $\mathrm{YH}$, Chou SH, Liang SW, Ni CE, Lin YT, Huang YW, et al. Emergence of an XDR and carbapenemase-producing hypervirulent Klebsiella pneumoniae strain in Taiwan. J Antimicrob Chemother. 2018;73:2039-46.

14. Leangapichart T, Lunha K, Jiwakanon J, Angkititrakul S, Jarhult JD, Magnusson U, et al. Characterization of Klebsiella pneumoniae complex isolates from pigs and humans in farms in Thailand: population genomic structure, antibiotic resistance and virulence genes. J Antimicrob Chemother. 2021;76:2012-6.

15. Liu C, Du P, Xiao N, Ji F, Russo TA, Guo J. Hypervirulent Klebsiella pneumoniae is emerging as an increasingly prevalent K. pneumoniae pathotype responsible for nosocomial and healthcare-associated infections in Beijing, China. Virulence. 2020;11:1215-24.

16. Siu LK, Fung CP, Chang FY, Lee N, Yeh KM, Koh TH, et al. Molecular typing and virulence analysis of serotype K1 Klebsiella pneumoniae strains isolated from liver abscess patients and stool samples from noninfectious subjects in Hong Kong, Singapore, and Taiwan. J Clin Microbiol. 2011;49:3761-5.

17. Lin JC, Koh TH, Lee N, Fung CP, Chang FY, Tsai YK, et al. Genotypes and virulence in serotype K2 Klebsiella pneumoniae from liver abscess and non-infectious carriers in Hong Kong, Singapore and Taiwan. Gut Pathog. 2014;6:21.

18. Qu TT, Zhou JC, Jiang Y, Shi KR, Li B, Shen P, et al. Clinical and microbiological characteristics of Klebsiella pneumoniae liver abscess in East China. BMC Infect Dis. 2015:15:161.

19. Guo Y, Wang S, Zhan L, Jin Y, Duan J, Hao Z, et al. Microbiological and clinical characteristics of hypermucoviscous Klebsiella pneumoniae isolates associated with invasive infections in China. Front Cell Infect Microbiol. 2017;7:24.

20. Shi Q, Lan P, Huang D, Hua X, Jiang Y, Zhou J, et al. Diversity of virulence level phenotype of hypervirulent Klebsiella pneumoniae from different sequence type lineage. BMC Microbiol. 2018;18:94.

21. Fu Y, Xu M, Liu Y, Li A, Zhou J. Virulence and genomic features of a bla $a_{C T X-}$ M-3 and bla $a_{\text {CTX-M-14 }}$ coharboring hypermucoviscous Klebsiella pneumoniae of serotype K2 and ST65. Infect Drug Resist. 2019;12:145-59.

22. Hirai J, Sakanashi D, Kinjo T, Haranaga S, Fujita J. The first case of community-acquired pneumonia due to capsular genotype K2-ST86 hypervirulent Klebsiella pneumoniae in Okinawa, Japan: a case report and literature review. Infect Drug Resist. 2020;13:2237-43.

23. Liu Y, Long D, Xiang TX, Du FL, Wei DD, Wan LG, et al. Whole genome assembly and functional portrait of hypervirulent extensively drug-resistant NDM-1 and KPC-2 co-producing Klebsiella pneumoniae of capsular serotype K2 and ST86. J Antimicrob Chemother. 2019;74:1233-40.

24. Wei DD, Xiong XS, Mei YF, Du FL, Wan LG, Liu Y. Microbiological and clinical characteristics of Klebsiella pneumoniae isolates of K57 capsular serotype in China. Microb Drug Resist. 2021;27:391-400.

25. Kakuta N, Nakano R, Nakano A, Suzuki Y, Masui T, Horiuchi S, et al. Molecular characteristics of extended-spectrum beta-lactamase-producing Klebsiella pneumoniae in Japan: predominance of CTX-M-15 and emergence of hypervirulent clones. Int J Infect Dis. 2020;98:281-6. 
26. Ernst CM, Braxton JR, Rodriguez-Osorio CA, Zagieboylo AP, Li L, Pironti A, et al. Adaptive evolution of virulence and persistence in carbapenemresistant Klebsiella pneumoniae. Nat Med. 2020;26:705-11.

27. Cubero M, Marti S, Dominguez MA, Gonzalez-Diaz A, Berbel D, Ardanuy C. Hypervirulent Klebsiella pneumoniae serotype K1 clinical isolates form robust biofilms at the air-liquid interface. PLoS One. 2019;14:e0222628.

28. Li G, Shi J, Zhao Y, Xie Y, Tang Y, Jiang X, et al. Identification of hypervirulent Klebsiella pneumoniae isolates using the string test in combination with Galleria mellonella infectivity. Eur J Clin Microbiol Infect Dis. 2020;39:1673-9.

29. Zhou K, Tang X, Wang L, Guo Z, Xiao S, Wang Q, et al. An emerging clone (ST457) of Acinetobacter baumannii clonal complex 92 with enhanced virulence and increasing endemicity in South China. Clin Infect Dis. 2018;67:S179-s188.

30. Clinical and laboratory standards institute (CLSI). The performance stands for antimicrobial susceptibility testing 2020:The M100-S130.

31. Li J, Zou M, Dou Q, Hu Y, Wang H, Yan Q, et al. Characterization of clinical extensively drug-resistant Pseudomonas aeruginosa in the Hunan province of China. Ann Clin Microbiol Antimicrob. 2016;15:35.

32. Li J, Huang ZY, Yu T, Tao XY, Hu YM, Wang HC, et al. Isolation and characterization of a sequence type 25 carbapenem-resistant hypervirulent Klebsiella pneumoniae from the mid-south region of China. BMC Microbiol. 2019;19:219.

33. Coffey BM, Anderson GG. Biofilm formation in the 96-well microtiter plate. Methods Mol Biol. 2014;1149:631-41.

34. Devanga Ragupathi NK, Muthuirulandi Sethuvel DP, Triplicane Dwarakanathan H, Murugan D, Umashankar Y, Monk PN, et al. The influence of biofilms on carbapenem susceptibility and patient outcome in device associated K. pneumoniae infections: insights into phenotype vs genomewide analysis and correlation. Front Microbiol. 2020;11:591679.

35. Ruan Z, Feng Y. BacWGSTdb, a database for genotyping and source tracking bacterial pathogens. Nucleic Acids Res. 2016;44:D682-7.

\section{Publisher's Note}

Springer Nature remains neutral with regard to jurisdictional claims in published maps and institutional affiliations.

Ready to submit your research? Choose BMC and benefit from:

- fast, convenient online submission

- thorough peer review by experienced researchers in your field

- rapid publication on acceptance

- support for research data, including large and complex data types

- gold Open Access which fosters wider collaboration and increased citations

- maximum visibility for your research: over $100 \mathrm{M}$ website views per year

At BMC, research is always in progress.

Learn more biomedcentral.com/submissions 